\title{
Editorial: Mitochondrial Remodeling and Dynamic Inter-Organellar Contacts in Cardiovascular Physiopathology
}

\begin{abstract}
Gaetano Santulli ${ }^{1,2,3}$, Giovanni Monaco ${ }^{4,5}$, Valentina Parra ${ }^{6,7,8}$ and Giampaolo Morciano ${ }^{9,10 *}$
${ }^{1}$ Division of Cardiology, Department of Medicine, Albert Einstein College of Medicine, Wilf Family Cardiovascular Research Institute and Einstein Institute for Aging Research, New York, NY, United States, ${ }^{2}$ Department of Molecular Pharmacology, Einstein-Sinai Diabetes Research Center (ES-DRC), Fleischer Institute for Diabetes and Metabolism (FIDAM), Montefiore University Hospital, New York, NY, United States, ${ }^{3}$ International Translational Research and Medical Education Academic Research Unit (ITME), Department of Advanced Biomedical Sciences, "Federico II" University, Naples, Italy, ${ }^{4}$ Center for Innovation and Stimulation of Drug Discovery (CISTIM), Leuven, Belgium, ${ }^{5}$ Laboratory of Molecular and Cellular Signaling, Department of Cellular and Molecular Medicine, KU Leuven, Leuven, Belgium, ${ }^{6}$ Departamento de Bioquímica y Biología Molecular, Facultad de Ciencias Químicas y Farmacéuticas, Universidad de Chile, Santiago, Chile, ${ }^{7}$ Advanced Center of Chronic Diseases (ACCDiS), Facultad de Ciencias Químicas y Farmacéuticas y Facultad de Medicina, Universidad de Chile, Santiago, Chile, ${ }^{8}$ Network for the Study of High-Lethality Cardiopulmonary Diseases (REECPAL), Universidad de Chile, Santiago, Chile, ${ }^{9}$ Department of Medical Sciences, Section of Experimental Medicine, Laboratory for Technologies of Advanced Therapies (LTTA), University of Ferrara, Ferrara, Italy, ${ }^{10}$ Maria Cecilia Hospital, GVM Care and Research, Cotignola, Italy
\end{abstract}

Keywords: cardiovascular disease, mitochondria, sarcoplasmic reticulum, contact sites, signal transduction

Editorial on the Research Topic

Mitochondrial Remodeling and Dynamic Inter-Organellar Contacts in
Cardiovascular Physiopathology

University of California, Davis, United States

*Correspondence: Giampaolo Morciano mrcgp/@unife.it; gmorciano@gvmnet.it

Specialty section:

This article was submitted to Cellular Biochemistry,

a section of the journa Frontiers in Cell and Developmental Biology

Received: 12 March 2021 Accepted: 31 March 2021 Published: 30 April 2021

Citation: Santulli G, Monaco G, Parra V and Morciano G (2021) Editorial: Mitochondrial Remodeling and Dynamic Inter-Organellar Contacts in Cardiovascular Physiopathology. Front. Cell Dev. Biol. 9:679725. doi: 10.3389/fcell.2021.679725
Emerging evidence has shown that membranes of many subcellular organelles are dynamic and engage in structural and functional communications, thereby creating new intracellular compartments by either sharing proteins or by owning a distinct pool (Rizzuto et al., 1998; Giorgi et al., 2018).

Membranes from different organelles do not fuse together, but preserve their integrity by approaching not more than a few nanometers (usually $10 \mathrm{~nm}$ ); this distance is enough to create transient contacts which significantly impact physiological processes (e.g., lipid metabolism, material exchange) (Simmen and Tagaya, 2017; Vance, 2020) and human diseases (van Vliet and Agostinis, 2018; Simoes et al., 2020).

Growing advances in technologies, including cell fractionation (Wieckowski et al., 2009; Montesinos and Area-Gomez, 2020), confocal (Chung et al., 2015; Galmes et al., 2016), and transmission electron microscopy (Csordás et al., 2006), alongside new tools which combine biochemistry and online databases (e.g., Contact-ID) (Kwak et al., 2020), have allowed the study of contact sites in many types of living cells, in order to address new structural, functional, and modulatory properties.

Contact sites in cardiomyocytes, especially those established between sarcoplasmic reticulum (SR), and transverse tubules (TT) of the sarcolemma, and with mitochondrial membranes, are necessary for excitation-contraction coupling (ECC) efficiency (Gambardella et al., 2018) and suitable calcium signaling (Fearnley et al., 2011). The latter sustains cell survival by modulating mitochondrial ATP generation to match cardiac workload and also cell death (Jouaville et al., 1999; Traaseth et al., 2004; Bonora et al., 2019). 
Among the intracellular organelles, mitochondria play an essential role in cardiomyocyte bioenergetics, because they constitute $35 \%$ of the total cell volume to satisfy the high-energy demand of heart (Elfering et al., 2004; Benard et al., 2007). As such, it is not surprising that mitochondrial dysfunction underlies several defects observed during heart development and differentiation, participating actively in the pathogenesis of a number of cardiovascular diseases (Santulli et al., 2015; BravoSagua et al., 2020). Hence, maintaining a healthy mitochondrial population is an essential homeostatic requirement that the cell retains by controlling multiple checkpoints including a balanced ratio between mitophagy and biogenesis, including mitochondrial fission and fusion (Morciano et al., 2020).

The present collection includes 11 reports subdivided in the following categories: basic mechanisms, human diseases, and therapies.

\section{BASIC MECHANISMS}

Five out of 11 reports belong to this category and are authored by Rossini and Filadi, Lin et al., Gilkerson et al., Lynch et al., and Piquereau et al.. The authors highlighted the importance of the cytoarchitecture, especially SR-mitochondria contact sites and spatio-temporal mitochondrial remodeling, in some molecular pathways essential for cardiomyocyte function. These include calcium signaling, one of the main players in mitochondrial bioenergetics and cardiac contractility; in this context, organelles and proteins involved in intracellular calcium fluxes have been analyzed both in vitro and in vivo. Moreover, new insights have been provided about reactive oxygen species (ROS) production, mitochondrial dynamics, and quality control in the adaptation of the heart to multiple stress conditions. Lastly, there is a report highlighting the ability of sex hormones as factors able to influence metabolism via mitochondrial remodeling.

\section{DISEASES}

Four manuscripts authored by Gao et al., Salazar-Ramírez et al., Ramaccini et al., and Kumar et al. report compelling evidence of how mitochondrial dysfunction and alterations in organelle communication can impact cellular homeostasis in cardiovascular diseases. Indeed, the rewiring of calcium signaling at SR-mitochondria interface (but also at the sarcolemma), the imbalance in mitophagy, defects in fusionfission machinery, lipid biosynthesis, ATP and ROS production are analyzed in a wide range of pathologies including dilated cardiomyopathy (DCM), heart failure, ischemia-reperfusion injury, and cardiac arrythmia.

\section{REFERENCES}

Benard, G., Bellance, N., James, D., Parrone, P., Fernandez, H., Letellier, T., et al. (2007). Mitochondrial bioenergetics and structural network organization. J. Cell Sci. 120, 838-848. doi: 10.1242/jcs.03381

Bonora, M., Wieckowski, M. R., Sinclair, D. A., Kroemer, G., Pinton, P., and Galluzzi, L. (2019). Targeting mitochondria for cardiovascular

\section{THERAPIES}

In CVD, the altered mitochondrial remodeling and impaired inter-organellar communications of cardiomyocytes may be amenable to therapeutic interventions, especially considering the dynamic and reversible nature of these interactions (Ferrandi et al., 2013; Sabbah, 2016; Siasos et al., 2018; Kerkhofs et al., 2019). In this sense, the last 2 reports authored by Elorza et al. and Angebault et al. summarize the currently available therapies targeting mitochondrial fitness (e.g., maintaining the correct balance of biogenesis and the control of mitochondrial heteroplasmy to prevent age-related diseases) and report the beneficial effects of metformin in mice affected by Duchenne muscular dystrophy (DMD)-associated cardiomyopathy. In this preclinical model, metformin was able to normalize SR-mitochondria interactions, and restore the function of the electron transport chain (ETC) Complex I and the expression of mitochondrial calcium-handling protein complexes.

\section{AUTHOR CONTRIBUTIONS}

GS and GMor conceived, wrote, and finalized the Editorial. GMon and VP wrote the Editorial.

\section{FUNDING}

The Santulli Lab was supported in part by the National Institutes of Health (NIH: R01-DK123259, R01-HL146691, R01DK033823, R01-HL159062, R00-DK107895, R56-AG066431, T32-HL144456 to GS), and by the Irma T. Hirschl and Monique Weill-Caulier Trusts (to GS). VP was supported by grants from the Fondo Nacional de Desarrollo Científico y Tecnológico, FONDECYT, Chile (1190743), Fondo de Financiamiento de Centros de Investigación en Áreas Prioritarias, FONDAP, Chile (15130011), International Centre for Genetic Engineering and Biotechnology, ICGEB, Italy (CRP-ICGEB Research Grant CRP/CHL18-04), and U-Redes Generación, Vicerrectoría de Investigación y Desarrollo, Universidad de Chile, Chile (G_201835). GM was supported by the Italian Ministry of Health Grant (GR-2019-12369862).

\section{ACKNOWLEDGMENTS}

We thank Ana María Avalos for proofreading and revising our manuscript.

disorders: therapeutic potential and obstacles. Nat. Rev. Cardiol. 16, 33-55. doi: 10.1038/s41569-018-0074-0

Bravo-Sagua, R., Parra, V., Muñoz-Cordova, F., Sanchez-Aguilera, P., Garrido, V., Contreras-Ferrat, A., et al. (2020). Sarcoplasmic reticulum and calcium signaling in muscle cells: homeostasis and disease. Int. Rev. Cell Mol. Biol. 350, 197-264. doi: 10.1016/bs.ircmb.2019. 12.007 
Chung, J., Torta, F., Masai, K., Lucast, L., Czapla, H., Tanner, L. B., et al. (2015). Intracellular transport. PI4P/phosphatidylserine countertransport at ORP5and ORP8-mediated ER-plasma membrane contacts. Science 349, 428-432. doi: 10.1126/science.aab1370

Csordás, G., Renken, C., Várnai, P., Walter, L., Weaver, D., Buttle, K. F., et al. (2006). Structural and functional features and significance of the physical linkage between ER and mitochondria. J. Cell Biol. 174, 915-921. doi: $10.1083 /$ jcb.200604016

Elfering, S. L., Haynes, V. L., Traaseth, N. J., Ettl, A., and Giulivi, C. (2004). Aspects, mechanism, and biological relevance of mitochondrial protein nitration sustained by mitochondrial nitric oxide synthase. Am. J. Physiol. Heart Circ. Physiol. 286, H22-H29. doi: 10.1152/ajpheart.00766. 2003

Fearnley, C. J., Roderick, H. L., and Bootman, M. D. (2011). Calcium signaling in cardiac myocytes. Cold Spring Harb. Perspect. Biol. 3:a004242. doi: $10.1101 /$ cshperspect.a004242

Ferrandi, M., Barassi, P., Tadini-Buoninsegni, F., Bartolommei, G., Molinari, I., Tripodi, M. G., et al. (2013). Istaroxime stimulates SERCA2a and accelerates calcium cycling in heart failure by relieving phospholamban inhibition. $\mathrm{Br}$. J. Pharmacol. 169, 1849-1861. doi: 10.1111/bph.12278

Galmes, R., Houcine, A., van Vliet, A. R., Agostinis, P., Jackson, C. L., and Giordano, F. (2016). ORP5/ORP8 localize to endoplasmic reticulummitochondria contacts and are involved in mitochondrial function. EMBO Rep. 17, 800-810. doi: 10.15252/embr.201541108

Gambardella, J., Trimarco, B., Iaccarino, G., and Santulli, G. (2018). New insights in cardiac calcium handling and excitation-contraction coupling. Adv. Exp. Med. Biol. 1067, 373-385. doi: 10.1007/5584_2017_106

Giorgi, C., Danese, A., Missiroli, S., Patergnani, S., and Pinton, P. (2018). Calcium dynamics as a machine for decoding signals. Trends Cell Biol. 28, 258-273. doi: 10.1016/j.tcb.2018.01.002

Jouaville, L. S., Pinton, P., Bastianutto, C., Rutter, G. A., and Rizzuto, R. (1999). Regulation of mitochondrial ATP synthesis by calcium: evidence for a longterm metabolic priming. Proc. Natl. Acad. Sci. U.S.A. 96, 13807-13812. doi: 10.1073/pnas.96.24.13807

Kerkhofs, M., Bultynck, G., Vervliet, T., and Monaco, G. (2019). Therapeutic implications of novel peptides targeting ER-mitochondria Ca2+-flux systems. Drug Discov. Today 24, 1092-1103. doi: 10.1016/j.drudis.2019.03.020

Kwak, C., Shin, S., Park, J.-S., Jung, M., Nhung, T. T. M., Kang, M.-G., et al. (2020). Contact-ID, a tool for profiling organelle contact sites, reveals regulatory proteins of mitochondrial-associated membrane formation. Proc. Natl. Acad. Sci. U.S.A. 117, 12109-12120. doi: 10.1073/pnas.1916584117

Montesinos, J., and Area-Gomez, E. (2020). Isolation of mitochondria-associated ER membranes. Methods Cell Biol. 155, 33-44. doi: 10.1016/bs.mcb.2019.12.001

Morciano, G., Patergnani, S., Bonora, M., Pedriali, G., Tarocco, A., Bouhamida, E., et al. (2020). Mitophagy in cardiovascular diseases. J. Clin. Med. 9:892. doi: $10.3390 /$ jcm 9030892
Rizzuto, R., Pinton, P., Carrington, W., Fay, F. S., Fogarty, K. E., Lifshitz, L. M., et al. (1998). Close contacts with the endoplasmic reticulum as determinants of mitochondrial $\mathrm{Ca} 2+$ responses. Science 280, 1763-1766. doi: $10.1126 /$ science. 280.5370 .1763

Sabbah, H. N. (2016). Targeting mitochondrial dysfunction in the treatment of heart failure. Expert Rev. Cardiovasc. Ther. 14, 1305-1313. doi: 10.1080/14779072.2016.1249466

Santulli, G., Xie, W., Reiken, S. R., and Marks, A. R. (2015). Mitochondrial calcium overload is a key determinant in heart failure. Proc. Natl. Acad. Sci. U.S.A. 112, 11389-11394. doi: 10.1073/pnas.1513047112

Siasos, G., Tsigkou, V., Kosmopoulos, M., Theodosiadis, D., Simantiris, S., Tagkou, N. M., et al. (2018). Mitochondria and cardiovascular diseases-from pathophysiology to treatment. Ann. Transl. Med. 6:256. doi: 10.21037/atm.2018.06.21

Simmen, T., and Tagaya, M. (2017). Organelle communication at Membrane Contact Sites (MCS): from curiosity to center stage in cell biology and biomedical research. Adv. Exp. Med. Biol. 997, 1-12. doi: 10.1007/978-981-10-4567-7_1

Simoes, I. C. M., Morciano, G., Lebiedzinska-Arciszewska, M., Aguiari, G., Pinton, P., Potes, Y., et al. (2020). The mystery of mitochondria-ER contact sites in physiology and pathology: a cancer perspective. Biochim. Biophys. Acta Mol. Basis Dis. 1866:165834. doi: 10.1016/j.bbadis.2020.165834

Traaseth, N., Elfering, S., Solien, J., Haynes, V., and Giulivi, C. (2004). Role of calcium signaling in the activation of mitochondrial nitric oxide synthase and citric acid cycle. Biochim. Biophys. Acta 1658, 64-71. doi: 10.1016/j.bbabio.2004.04.015

van Vliet, A. R., and Agostinis, P. (2018). Mitochondria-associated membranes and ER stress. Curr. Top. Microbiol.Immunol. 414, 73-102.doi: 10.1007/82_2017_2

Vance, J. E. (2020). Inter-organelle membrane contact sites: implications for lipid metabolism. Biol. Direct. 15:24. doi: 10.1186/s13062-020-00279-y

Wieckowski, M. R., Giorgi, C., Lebiedzinska, M., Duszynski, J., and Pinton, P. (2009). Isolation of mitochondria-associated membranes and mitochondria from animal tissues and cells. Nat. Protoc. 4, 1582-1590.s doi: $10.1038 /$ nprot.2009.151

Conflict of Interest: The authors declare that the research was conducted in the absence of any commercial or financial relationships that could be construed as a potential conflict of interest.

Copyright (c) 2021 Santulli, Monaco, Parra and Morciano. This is an open-access article distributed under the terms of the Creative Commons Attribution License (CC $B Y)$. The use, distribution or reproduction in other forums is permitted, provided the original author(s) and the copyright owner(s) are credited and that the original publication in this journal is cited, in accordance with accepted academic practice. No use, distribution or reproduction is permitted which does not comply with these terms. 